\title{
Negotiating on Location, Timing, Duration, and Participant in Agent-Mediated Joint Activity-Travel Scheduling
}

Huiye Ma

Faculty of Architecture, Building and Planning

Eindhoven University of Technology

P.O. Box 513 - 5600 MB Eindhoven

The Netherlands

Tel: +31 402474527

Fax: +31402438488

E-mail: H.Ma@tue.nl

Nicole Ronald

Faculty of Architecture, Building and Planning

Eindhoven University of Technology

P.O. Box 513 - 5600 MB Eindhoven

The Netherlands

Tel: +31 402472934

Fax: +31402438488

E-mail: N.A.Ronald@tue.nl

Theo A. Arentze

Faculty of Architecture, Building and Planning

Eindhoven University of Technology

P.O. Box 513 - 5600 MB Eindhoven

The Netherlands

Tel: +31 402472283

Fax: +31 402438488

E-mail: T.A.Arentze@tue.nl

Harry J. P. Timmermans

Faculty of Architecture, Building and Planning

Eindhoven University of Technology 
P.O. Box 513 - 5600 MB Eindhoven

The Netherlands

Tel: +31 402472274

Fax: +31 402438488

E-mail: H.J.P.Timmermans@tue.nl

\section{Negotiating on Location, Timing, Duration, and Participant in Agent-Mediated Joint Activity-Travel Scheduling}

\footnotetext{
Abstract

Agent-based simulation has become an important modeling approach in activity-travel analysis. Social activities account for a large amount of travel and have an important effect on activitytravel scheduling. Participants in joint activities usually have various options regarding location, participants, and timing, and take different approaches to make their decisions. In this context, joint activity participation requires negotiation among agents involved so that conflicts among the agents can be addressed. Existing mechanisms do not fully provide a solution when utility functions of agents are nonlinear and non-monotonic. Considering activity-travel scheduling in time and space as an application, we propose a novel negotiation approach, which takes into account these properties, such as continuous and discrete issues, nonlinear and non-monotonic utility functions, by defining a concession strategy and a search mechanism. The results of experiments show that agents having these properties can negotiate efficiently. Furthermore, the negotiation procedure affects individuals' choices of location, timing, duration, and participants.

Keywords

Negotiation, nonlinearity, activity-travel scheduling, joint activity participation, multi-agent simulation
} 


\section{Introduction}

Existing approaches to the modeling of joint activities in activity-based analysis, as this field has developed in geography, urban planning, and transportation research, focus on households while virtually ignoring the larger social networks of individuals. Household joint activities have been predominantly modeled in terms of discrete choice models, assuming explicitly or implicitly complete information settings (e.g., Scott et al. 2002, Gliebe et al. 2002; Zhang et al. 2005; Kuwano et al. 2011).

In fact, social activities account for a large amount of travel (e.g., Van den Berg et al. 2012) and have an important effect on activity-travel scheduling. Modeling activity scheduling in social groups offers a challenge because participants usually have various options regarding location, participants, and timing, and take different approaches to make their decisions. Ronald et al. (2009) have assumed a host, who acts as the initiator, processes all the members' information, and eventually makes a decision for all. Therefore, the environment is fully cooperative in that all the agents "vote" for one agent as an administrator who will collect the proposals from all the agents and later make a fair decision on the joint activity among these agents. Such an approach seems especially appropriate in situations of mutual agreements on joint activities and the host being well aware of the utility of each participant. Fang et al. (2011) have described a multi-objective approach for scheduling joint participation by adopting the non-dominated sorting genetic algorithm-II (NSGA-II) with multiple objectives. In this approach, the preferences of all participants are known in advance and are used to find optimal opportunities. Neutens et al. (2010) have made similar assumptions because they require knowledge of the time constraints for all the participants, and the utility function of the group.

However, these conditions may not be met in social networks of acquaintances, where there may not be a single participant to command all; and utility functions or preferences or time constraints may be private to each participant. In these situations, the environment becomes semi-cooperative in that participants are trying to satisfy their needs whilst compromising to others' needs.

Under these circumstances, a process of coordination and possible conflict resolution is therefore required. This process has some essential features in common with negotiation in agent-based systems, which can be described as a "distributed search through a space of potential agreements" (Jennings et al. 2001). It involves defining the issues on which an agreement needs to be made (e.g., where to meet, at what time, who is invited to the activity), the rules of the interaction (i.e., the exchange of information and choices), and how agents move towards an agreement (e.g., if the agents do not agree, some of them will make a concession by suggesting different proposals, such as a different location, or a shorter duration, or a later start time etc.). This approach can be used at different temporal and geographical scales; is not restricted to household members; and can take a dynamic environment into account. 
Although some work has been previously carried out in semi-cooperative environments (e.g., Ren et al. 2010), it has been restricted to one issue and two players. In activity-travel scheduling, however, negotiation about joint activities involves usually multiple issues, such as the time to start a joint activity, the duration of the activity, where to conduct the activity, and with whom to undertake the activity. Some of these issues involve discrete choices, such as location and participants, while other choices are continuous, such as duration. Moreover these issues may be interrelated and therefore the utility function could be nonlinear.

Before undertaking any empirical analyses, it is important to examine the face validity of the model. Numerical simulations are valuable in that context as they allow investigating whether the formulated model is in line with one's theoretical expectations. As a start, we focus on small scale computer simulations so as to better understand strategic behaviors, such as compromising to others; and collect preliminary results about the performance of the model.

Thus, in this paper, we present a negotiation protocol in agent-mediated joint activity-travel scheduling. We develop a negotiation approach to cater for the problems inherent in semicooperative environments with nonlinear, non-monotonic, and continuous utility functions involving several issues and multiple players. Results of simulations show that the negotiators do reach an agreement by following the protocol. Section 2 discusses related work. Section 3 outlines the proposed negotiation model with a concession strategy. In Section 4, the results of an illustrative experiment are given in order to demonstrate the performance of our proposed negotiation protocol. Finally, Section 5 concludes and discusses further work.

\section{Related work}

Negotiation has been extensively studied over the last decades and most research work has been conducted in fields such as social psychology, game theory, and artificial intelligence (Jennings et al. 2001; Osborne et al. 1994). One crucial feature of a negotiation process is information. In most realistic cases agents have incomplete information about their opponent(s) (Fatima et al. 2004). Game theory is compelling as a tool for negotiation in cases where it is possible to characterize the preferences and possible strategies of participants (Jennings et al. 2001). Game theoretic models have been proposed for bargaining with incomplete information (Avineri 2006; Hollander 2006). For instance, Rubinstein (Rubinstein 1985) has developed a model in which agents have incomplete information about time preferences. Sandholm and Vulkan (1999) have considered uncertainty about agent deadlines. All these models are built on the assumption that information about some uncertain parameter (in the form of possible values and a corresponding probability distribution) is part of the common knowledge of agents.

A major limitation of these game-theoretic methods is that the equilibrium solutions are difficult to apply in practice, especially in negotiation with incomplete information, or private knowledge, or non-linear utility functions (e.g. Jennings et al. 2001; Wu et al. 2009). For example, game 
theoretic models often assume perfect computational rationality which means that no computation is required to find mutually acceptable solutions within a feasible range of outcomes. Moreover, this space of possible agreements is often assumed to be fully known by the agents. This assumption is rarely true in real world cases because agents typically know their own information space, but they can hardly know that of their opponent(s). Furthermore, even if the joint space is known, knowing that a solution exists is entirely different to knowing what the solution actually is.

Heuristic methods are the key means of overcoming the aforementioned limitations of game theoretic models. Heuristic methods aim to produce good, rather than necessarily optimal solutions. The methods themselves may either be computational approximations of game theoretic techniques or computational realizations of more informal negotiation models (Jennings et al. 2001).

In our case, an agent's utility function and his beliefs about other agents are not known to other agents. We therefore treat each agent's information as private knowledge. Moreover, the scheduling of joint activities involves various issues. Some of these issues have discrete choices while others have continuous choices. One issue may be interrelated with other issue(s) as well. The outcome of evaluating these different issues becomes non-linear. Therefore in this work, we propose heuristic computational methods of negotiation strategies for agents with private knowledge and nonlinear utility functions. Work that falls in this category is discussed below.

Wainer et al. (2007) have described several protocols for scheduling a meeting with many participants. There is only one issue under discussion, the timing of the meeting. Several protocols are proposed which use different levels of privacy for the participants. Nevertheless, it is limited by the number of issues under discussion.

Ito et al. (2007) have presented a protocol for interdependent multiple issues with nonlinear utility. Their process involves sampling the issue space and then adjusting to find local maxima. Agents then submit their best options and a mediator determines the best solution. The mediator assumed by them is unrealistic from our perspective.

Wu et al. (2009) have described a negotiation protocol for multi-player multi-issue negotiations with incomplete information and presented results for a simulation with three agents and two issues. Again, the issues are assumed to be continuously-valued, and the utility function is assumed to be monotonically increasing. This has been extended by Ma et al. (2010) to include both discrete and continuous issues but it is still restricted by imposing certain forms of utility functions because the orthogonal strategy assumed requires either concave or convex curves.

Ren et al. (2010) have presented a bilateral single-issue negotiation model, focusing on handling non-linear utility functions and demonstrated the model in a situation involving a dental patient 
wanting to make an appointment. This work is inappropriate for our situation as it handles only two players with one discrete issue.

Rindt et al. (2003) have reported on the development of a simulation kernel for agent-based activity microsimulation built on the re-characterization of human activity as interaction between autonomous entities. They start from the idea that "human activity is the negotiated interaction of socially and physically situated individuals and settings" and as a result their kernel assumes that behavior is adaptive. The framework described is flexible and does not impose many restrictions on negotiations, but it is not a complete model in itself.

Given the wide variety of possibilities in different fields/problems, it should be clear that there is no universally best approach or technique for negotiation (Jennings et al. 2001). A systematic treatment of the negotiation cases involved in activity travel scheduling is still lacking.

\section{The Negotiation Model}

In this negotiation model, we assume agents, $1, \ldots, N$, are negotiating on four issues: start time of an activity, ST, location of the activity, LOC, participants of the activity, PAR, and the duration of the activity, DUR . Among these issues, ST, LOC, and PAR are discrete issues while DUR is a continuous issue.

\section{Utility Functions}

Given the activity travel scheduling issues, we assume that the proposed overall utility function for an offer $i$ by a given agent $a$ is defined as in Equation 1 (also see Figure 1).

$$
\mu_{\mathrm{a}}\left(\text { offer }_{\mathrm{i}}\right)=\beta_{\mathrm{a}}\left(\mathrm{ST}_{\mathrm{i}}, \mathrm{LOC}_{\mathrm{i}}, \mathrm{PAR}_{\mathrm{i}}\right) \times \ln \left(1+\mathrm{DUR}_{\mathrm{i}}\right)-\operatorname{oppcost}_{\mathrm{a}}\left(\mathrm{TT}_{\mathrm{i}, \mathrm{a}}, \mathrm{DUR}_{\mathrm{i}}\right)
$$

where offer $r_{i}=\left\{S T_{i}, L O C_{i}, P A R_{i}, D U R_{i}\right\} ; \mathrm{ST}_{\mathrm{i}}$ is the start time variable; $\mathrm{LOC}_{\mathrm{i}}$ is the location variable; $\mathrm{PAR}_{\mathrm{i}}$ is the participant variable; $\mathrm{DUR}_{\mathrm{i}}$ is the duration variable; $T T_{i, a}$ is the travel time to be spent for the joint activity at a certain location; and oppcost $t_{a}$ represents opportunity costs. In this formulation, beta represents an average utility of the activity per unit time. By multiplying the utility per unit time with the log of duration this is transformed into a total utility for the activity where the log transformation takes into account diminishing returns of time spent on the activity. In short, this formulation is consistent with economic theories of time use as first introduced by Becker et al. (1965).

In this utility function, $\mathrm{ST}_{\mathrm{i}}$ is handled as a discrete variable instead of a continuous variable. This reflects the idea that in the real world individuals often express preferences for time of an activity on the basis of a discrete set of episodes (e.g., late in the morning, early in the afternoon, etc.) rather than on exact times. 
$\mathrm{PAR}_{\mathrm{i}}$ tells which agent(s) will be considered in offer $i$ as participants. For example, if $\mathrm{PAR}_{\mathrm{i}}$ does not include agent $a$, then it means that according to the proposal the joint activity will be carried out by the other agent(s). In this case, the values of $\mathrm{ST}_{\mathrm{i}}, \mathrm{LOC}_{\mathrm{i}}, \mathrm{PAR}_{\mathrm{i}}, \mathrm{DUR}_{\mathrm{i}}$, and $T T_{i, a}$ do not make any sense to agent $a$. Then agent $a$ will let $\mathrm{DUR}_{\mathrm{i}}$ and $T T_{i, a}$ be equal to zero so that the resulting utility is also equal to zero. This reflects that, on the one hand, agent $a$ has no interest on such an activity as he is not included; on the other hand, agent $a$ has no interest in blocking such an activity for other agent(s). In this situation, the decision on such an activity will be made by the other agent(s).

According to the form of the utility function, if the utility of doing or following a joint activity is larger than or equal to the utility of the opportunity cost, then the overall utility $\mu_{\mathrm{a}}\left(\right.$ offer $\left._{\mathrm{i}}\right)$ is larger than or equal to zero and hence the agent is willing to carry out the joint activity or follow the decision made by other agent(s). If the value of $\mu_{\mathrm{a}}$ (offer $)$ is negative, then the agent will not consider the joint activity.

An example is shown in Figure 1 where the $\mathrm{X}$-axis represents the $\mathrm{DUR}_{\mathrm{i}}$ variable and the $\mathrm{Y}$-axis the overall utility of agent $a$. A unique combination of $\mathrm{ST}_{\mathrm{i}}, \mathrm{LOC}_{\mathrm{i}}, \mathrm{PAR}_{\mathrm{i}}, T T_{i, a}$ corresponds to a curve in the figure. It is also possible that a curve represents more than one combination that gives the agent the same utility outcome.

The first term on the RHS of Equation 1 is operationalized as follows:

$$
\beta_{a}\left(\mathrm{ST}_{\mathrm{i}}, \mathrm{LOC}_{\mathrm{i}}, \mathrm{PAR}_{\mathrm{i}}\right)=\beta_{S T, a}\left(\mathrm{ST}_{\mathrm{i}}\right) \times \beta_{L O C, a}\left(\mathrm{LOC}_{\mathrm{i}}\right) \times \beta_{P A R, a}\left(\mathrm{PAR}_{\mathrm{i}}\right)
$$

As an example, the utility function of the start time $\mathrm{ST}_{\mathrm{i}}$ can be defined as:

$$
\beta_{S T, a}\left(\mathrm{ST}_{\mathrm{i}}\right)= \begin{cases}-\infty & 0 \leq \mathrm{ST}_{\mathrm{i}}<10 \text { or } 22 \leq \mathrm{ST}_{\mathrm{i}}<24 \\ 0 & 18 \leq \mathrm{ST}_{\mathrm{i}}<20 \\ 2 & 10 \leq \mathrm{ST}_{\mathrm{i}}<12 \text { or } 16 \leq \mathrm{ST}_{\mathrm{i} i}<18 \text { or } 20 \leq \mathrm{ST}_{\mathrm{i}}<21 \\ 4 & 14 \leq \mathrm{ST}_{\mathrm{i}}<16 \\ 6 & 12 \leq \mathrm{ST}_{\mathrm{i}}<14 \text { or } 21 \leq \mathrm{ST}_{\mathrm{i}}<22\end{cases}
$$

In this example, agent $a$ prefers to do the activity between 12:00 and 14:00 or between 21:00 and 22:00 rather than at other times. Note that the discretization means that agents are not sensitive about whether the start time is 12:01 or 12:10. 
$\operatorname{oppcost}_{\mathrm{a}}\left(\mathrm{TT}_{\mathrm{i}, \mathrm{a}}, \mathrm{DUR}_{\mathrm{i}}\right)$ - the opportunity costs of undertaking a given activity - is operationally defined as follows:

$$
\operatorname{oppcost}_{\mathrm{a}}\left(\mathrm{TT}_{\mathrm{i}, \mathrm{a}}, \mathrm{DUR}_{\mathrm{i}}\right)=\omega_{a} \times\left(\mathrm{TT}_{\mathrm{i}, \mathrm{a}}+\mathrm{DUR}_{\mathrm{i}}\right)
$$

where $T T_{i, a}$ is the travel time to be spent for the joint activity at a certain location; $\omega_{a}$ represents a value of time. Assuming opportunity costs to be a linear function of travel time plus activity duration is based on the notion that time could be spent in many other ways and hence no diminishing returns occur on the level of opportunity costs.

The parameters involved in the utility function can be estimated by conducting stated choice experiments in which values of the parameters are systematically varied and hence the utility function can be estimated (Hensher et al. 2005). Examples of such stated choice experiments in travel behavior analysis include Nijland et al. (2011) and Grigolon et al. (2012). Compared with the analysis in the literature, there are several key features for the negotiation model:

- The overall utility function in Equation 1 is nonlinear, non-monotonic, and continuous. Our negotiation model can handle this and other types of utility functions as well.

- There are four main issues concerned in the negotiation, three discrete issues and one continuous issue. Our model can handle more discrete issues but at most one continuous issue.

- $\quad$ There can be more than two players involved in the negotiation, e.g., three or more.

\section{Multiple Offer Mechanism}

There are usually multiple offers conceivable which give the same amount of utility. Figure 1 shows an example where three offers $o f f e r_{a 1}$, offer $r_{22}$, and offer $r_{a 3}$ give the same value of utility, $U$. An agent is indifferent about these alternative offers because all of them have the same utility value to the agent. Therefore an agent is willing to submit these multiple offers together as a proposal. 


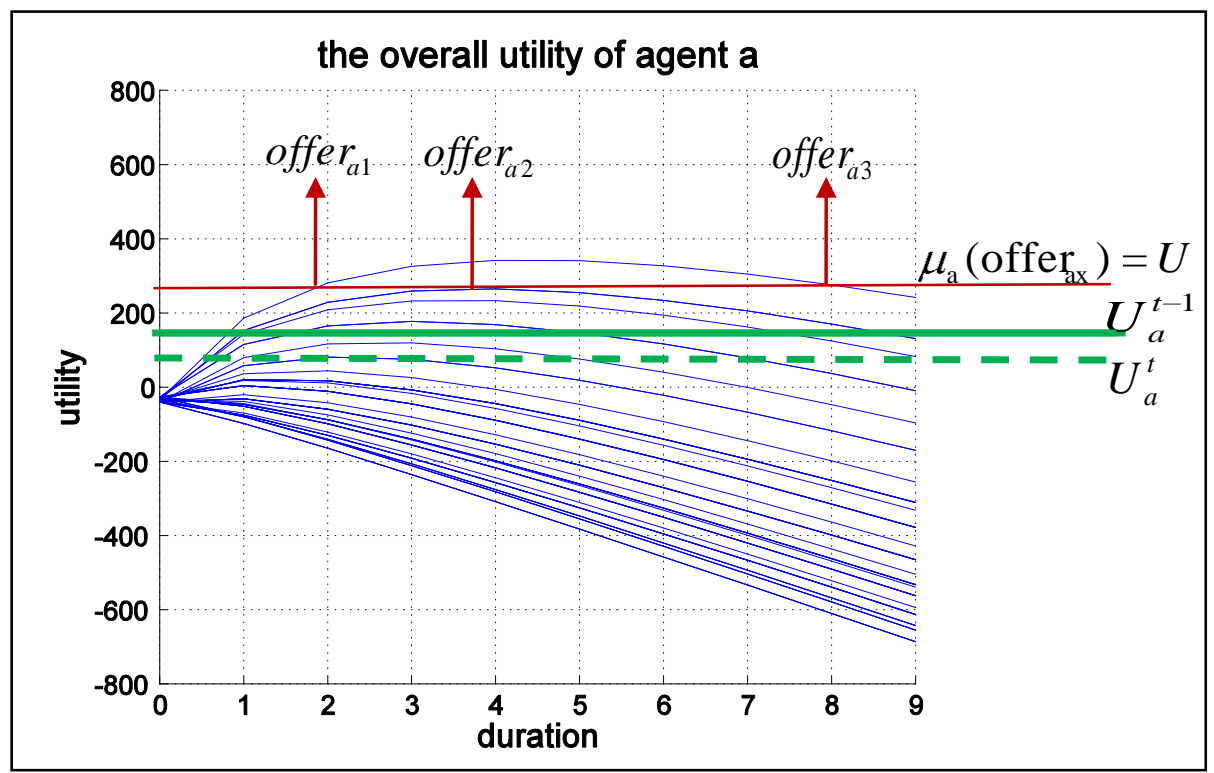

FIGURE 1. The overall utility function of agent a and an example of multiple offers (offer $r_{a 1}$, offer $r_{a 2}, o f f e r_{a 3}$ ) of agent $a$.

\section{Concession Strategy}

The negotiation takes place round by round until an agreement for all or some of the agents is reached or until some agent reaches his deadline. In each round, agents send their proposals sequentially and the order is generated randomly before the beginning of every round.

At any point in time, an agent assumes a so-called acceptable utility level to determine which offers he is willing to accept. A concession strategy is essential for an agent to decide the acceptable utility level that he is going to move to in the next round. The concession method depends on the information that an agent has at that moment.

- If there are no proposals in the history of the process, then the agent is the first one to give a proposal. Because there is no proposal from the history for the agent to judge or accept, the agent uses his own utility function to determine proposals which give him the highest utility. Therefore, no concession strategy is required for this case.

- If the agent is not the first one to give his proposal in the negotiation, then he can choose to concede by lowering his acceptance utility level by some amount. For example, in Figure 1 an agent $a$ concedes by setting $U_{a}^{t}=U_{a}^{t-1}-\delta$ where $\delta>0$ is a step size, $U_{a}^{t}$ is the acceptable utility level at time $t$ and $U_{a}^{t} \geq 0$. The bold line in green denotes the acceptable utility level of $U_{a}^{t-1}$ and the dashed line in green denotes that of $U_{a}^{t}$. The agent does not need to nor is allowed to know other agents' utility curves. 
- If the agent reaches the utility level equal to zero, then the agent cannot submit any new proposal(s) but he can still check if there is an agreement until all the agents come to utility of zero.

- If all the agents come to the utility equal to zero, it means that it is not possible for all the agents to reach an agreement. Then each agent will check if there are agreement(s) for some of the agents including himself. If there is such an agreement, then the agent will submit it to the other agents with the appropriate time stamp. The system will choose the one with the earliest time stamp as the final agreement for the agents involved.

Making a concession is not always the best choice for a rational agent. When there are some proposals submitted by other agents in the past, the agent can also decide to base his next move on previous proposals of others by moving towards their proposals, e.g., if the other agents have suggested an offer shared by them, SOffer $_{q}$, then the agent will compute his utility as if he would accept this offer, denoted as $\mu_{\mathrm{a}}\left(\mathrm{SOffer}_{\mathrm{q}}\right)$. If there is more than one shared offer, the agent computes all the utilities for him and identifies the best one denoted as SOffer $_{\mathrm{p}}$. A rational agent will compare which one gives him a higher utility. If the concession strategy gives him a higher utility, which means $\mu_{a}\left(\operatorname{SOffer}_{\mathrm{p}}\right)<U_{a}^{t}$, he will give his proposal according to the utility level $U_{a}^{t}$. Otherwise he will accept the proposal submitted by others before and consequently end the negotiation process.

\section{Model}

Each agent $\mathrm{a} \in[1, N]$ has a negotiation deadline $T_{a}$. A round is a moment when all the agents submit their proposals once. If the proposals can form an agreement in round $t$, the negotiation finishes successfully; otherwise the negotiation passes on to the next round. If no agreement is reached until the deadline, $T=\min \left\{T_{1}, \ldots, T_{N}\right\}$, the whole negotiation will stop unsuccessfully and every agent gets zero utility (i.e., the utility he gets from spending time in another way).

We use two agents $a$ and $b$ as an example to explain the following proposal generation process. Negotiation starts when the first proposal is made by an agent, say $a$. When agent $a$ receives a proposal from agent $b$ at time $t$, i.e., $\operatorname{Proposal}_{\mathrm{b}}(t)=\left(\ldots\right.$, offer $\left.r_{b p}, \ldots\right)$ which includes multiple offers but offer $_{b p}$ is the best choice for agent $a$, he evaluates the offer offer $_{b p}$ using his utility function $\mu_{a}\left(o f f e r_{b p}\right)$. If the value of $\mu_{a}\left(o f f e r_{b p}\right)$ at time $t$ is greater than or equal to the current acceptable utility level of $a$, i.e., $U_{a}^{t}$, then agent $a$ accepts offer $_{b p}$ submitted by agent $b$. Otherwise a counteroffer is made, denoted as $\left(\right.$ offer $r_{a 1}$, offer $r_{a 2}, \ldots$, offer $\left.r_{a s}\right)$, where $\mu_{a}\left(\right.$ offer $\left.r_{a x}\right)=U_{a}^{t} ; x$ is an integer and $\forall x \in[1, s]$; and $s$ is the number of possible offers where this equation holds. If the deadline $T$ is reached, then the negotiation ends and agent $a$ has to quit. 
We denote the agents, all or some, as a set $S A$ which includes the agents to be considered in an agreement. Thus, a proposal is formally defined as:

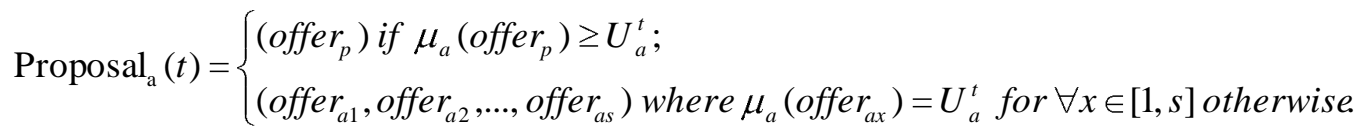

where as before, offer $r_{p}$ is one offer submitted by other agents $\in S A$ in the past and offer $r_{p}$ brings the highest utility to agent $a \in S A$ compared with other offers from other agents in the past; in fact, offer $_{p}$ is Shared Offer explained below; $t$ represents the current round; $a \in S A$ is an index of agent; $s$ is the total number of offers which meet the acceptance level utility $U_{a}^{t}$ of agent $a$ at time $t$.

Definition 1:

offer $r_{a l}=$ offer $_{b j}$ means that $S T_{a l}=S T_{b j}, L O C_{a l}=L O C_{b j}, P A R_{a l}=P A R_{b j}, D U R_{a l}=D U R_{b j}$ where offer $r_{a l}$ is submitted by agent $a \in S A$ and $o f f e r_{b j}$ is submitted by agent $b \in S A$.

Since proposals submitted by various agents in $S A$ are broadcast to each other, agent $a$ can keep a history of all these proposals by various agents in the negotiation.

Definition 2:

History $_{\text {all }}(\mathrm{t})=\left\{\operatorname{Proposal}_{\mathrm{o}}(y)\right\}$ where $\forall o \in S A ; \forall y \in[1, t]$.

Based on the history of all the proposals submitted by all the agents $\in S A$, History all $(\mathrm{t})$, agent $a$ is able to save all the proposals by the agents except agent $a$ himself into History $(\mathrm{t})$.

Definition 3:

History $_{\mathrm{a}}(\mathrm{t})=\left\{\operatorname{Proposal}_{\mathrm{r}}(y)\right\}$ where $a \in S A ; \forall r \in S A$ and $r \neq a ; \forall y \in[1, t]$.

Suppose there are $M$ agents in $S A$ and $M \leq N$. According to History $(\mathrm{t})$, agent $a$ can further compare all the proposals by the other agents. When there are different moments $t_{1}, t_{2}, \ldots$, $t_{M-1} \in[1, t] \quad$ such that $\operatorname{Proposal}_{r_{1}}\left(t_{1}\right) \cap \operatorname{Proposal}_{r_{2}}\left(t_{2}\right) \cap \ldots \cap \operatorname{Proposal}_{r_{M-1}}\left(t_{M-1}\right)=S S O$ and $S S O \neq \varnothing$, it means that these proposals have one or more offers in common and agent $a$ will save these shared offers into SharedOffer $(t)$.

Definition 4:

SharedOffer $_{\mathrm{a}}(\mathrm{t})=\left\{\right.$ SOffer $\left._{\mathrm{c}}\right\}$ where SOffer $_{\mathrm{c}} \in S S O ; c \in[1, Z] ; \mathrm{Z}$ is the total number of SOffer in $S S O$.

Definition 5: 
An agreement is achieved by $M$ agents when there are $t_{1}, t_{2}, \ldots, t_{M} \in[1, t]$ such that $\operatorname{Proposal}_{1}\left(t_{1}\right) \cap \operatorname{Proposal}_{2}\left(t_{2}\right) \cap \ldots \cap \operatorname{Proposal}_{\mathrm{M}}\left(t_{M}\right) \neq \varnothing$.

\section{Search Mechanism}

The search for an agreement by an agent $a$ in one round is carried out in the following steps. If there is no agreement and the deadline is not reached yet in this round, then the search process will continue in the next round.

1. Agent $a$ calculates his acceptable utility level denoted as $U_{a}^{t}$ according to the agent's concession strategy at negotiation round $t$;

2. Based on all the available proposals in the history, History ${ }_{\text {all }}(\mathrm{t})$, the agent finds the offer(s) which are shared by the other agents, SharedOffer ${ }_{a}(t)$;

3. Agent $a$ computes $\mu_{\mathrm{a}}\left(\right.$ SOffer $\left._{\mathrm{p}}\right)$ where SOffer $_{\mathrm{p}}$ is the best shared offer for him.

4. The agent will accept SOffer only if $\mu_{\mathrm{a}}\left(\right.$ SOffer $\left._{\mathrm{p}}\right) \geq U_{a}^{t}$. Then there is an agreement and the search process ends.

5. Before the deadline $T$ is reached, if all agents come to utility equal to zero, agent $a$ will give up searching for an agreement of all the agents but start to look for an agreement of some agents.

a. Agent $a$ will denote the valid subset of all participants as part and find the offers shared by these participants, SharedOfferPart ${ }_{\mathrm{a}}(\mathrm{t})$;

b. Agent $a$ will repeat step 1 to 4 while replacing SharedOffer $(\mathrm{t})$ by SharedOfferPart ${ }_{\mathrm{a}}(\mathrm{t})$;

c. If there is still no agreement for part, then agent $a$ changes to another valid subset of all the participants and jumps to step 5.a.

6. If the deadline $T$ is reached, then there is no agreement and the search process ends.

\section{Protocol}

A negotiation protocol for $N$ agents taking into account the search mechanism is proposed below. A detailed procedure is shown in pseudo code in Figure 2 where Line 10 to Line 13 is the search for an agreement; Line 15 to Line 17 is to submit a proposal by the current agent; Line 21 to Line 29 is to search an agreement with fewer participants; Line 37 to Line 42 is to make a concession for all the agents after one round. Less formally, the protocol can be written as follows:

1. All agents assign negotiation parameters before a negotiation starts, i.e., his own utility function, his own negotiation deadline.

2. A round starts. 
3. One agent is randomly assigned to start making a proposal. The agent calculates all his acceptable offers according to the utility function and the current acceptance utility level.

4. If the existing shared offers by the other agents give equal or larger utility to the agent than his own counter-offer(s), then the negotiation is completed. If not and it is his deadline, then the negotiation is completed with no agreement. Otherwise the agent sends counter-offer(s) to the other agents. Then another randomly assigned agent will repeat Step 3 and step 4 until all the agents submit their counter-offers for the current round.

5. At the conclusion of a round, all negotiators calculate the concessions they are willing to make for the next round. Return to step 2 to commence a new round until all the agents come to utility of zero.

6. If there is an agreement for some of the agents, then an agent will submit it to the other agents with the appropriate time stamp. The system will choose the one with the earliest time stamp as the final agreement for the involved agents.

The concession process continues until there is an agreement, or the deadline is reached, or all acceptable utility levels have reached zero without an agreement. Therefore, if there is no mutual agreement at the end of the negotiation, the reason may be that the deadline is too soon for any agent to try all the possible proposals; or there is no acceptable mutual agreement among all or some of the agents. In other words, our proposed protocol can guarantee finding the agreement if the deadline is long enough and if there is at least one proposal which brings non-negative utility for all or some of the agents. If all or some of the agents do not reach a mutual agreement while deadlines are not restrictive, then negotiations are unsuccessful because there is in fact no solution even if all agents concede to a maximum extent. In case multiple agreements exist in the end, the system will choose one randomly because any of them satisfies the Pareto optimality requirement. 
1. There are $N$ agents; $T$ is the maximum round of the negotiation process;

2. History $_{\text {all }}(\mathrm{t})=\left\{\operatorname{Proposal}_{\mathrm{o}}(p)\right\}$ where $o \in[1, N] ; p \in[1, t] ; t \in[1, T]$;

3. $\mu_{0}^{\mathrm{t}}$ denotes the utility level of an agent $o$ in round $t ; t=1$; bSubmit=true;

4. while $(t \leq T)$ do

5. Randomly arrange the sequence of agents;

6. $\quad i=1$;

7. while $(i \leq N)$ do

8. $\quad$ Find the shared offers SharedOffer ${ }_{i}(t)$;

9. Find the shared offer best for agent $i$, $\operatorname{SOffer}_{\mathrm{p}}$, which satisfies $\mu_{i}\left(\operatorname{SOffer}_{\mathrm{p}}(\mathrm{t})\right) \geq \mu_{i}\left(\operatorname{SOffer}_{\mathrm{q}}(\mathrm{t})\right)$ where $\forall q \operatorname{SOffer}_{\mathrm{q}} \in \operatorname{SharedOffer}_{\mathrm{i}}(\mathrm{t})$ and $p \neq q ;$

10. $\quad$ if $\left(\mu_{\mathrm{i}}\left(\mathrm{SOffer}_{\mathrm{p}}\right) \geq U_{i}^{t}\right)$ then

11. $\quad$ agent $i$ accepts SOffer ;

12. there is an agreement for the agents;

13. break;

14. else

15. if (bSubmit==true)

16. submit his offers, $\left.\left(\text { offer } r_{1}, \text { offer } r_{2}, \ldots, \text { offer }\right)_{s}\right)$, which gives him the utility $U_{i}^{t}$;

17. end if

18. end if

19. if (all the agents come to zero utility)

20. // each agent goes to check agreements for less participants, but the more the happier.

21. Find the valid subset of participants denoted as part and find the offers shared by these participants, denoted as SharedOfferPart ${ }_{\mathrm{a}}(\mathrm{t})$;

$r=1$;

while $(r \leq T)$ do

Find the shared offer best for agent $i$, SOfferPart $\mathrm{p}_{\mathrm{p}}$, which satisfies $\mu_{i}\left(\right.$ SOfferPart $\left._{\mathrm{p}}(\mathrm{t})\right) \geq \mu_{i}\left(\right.$ SOfferPart $\left._{\mathrm{q}}(\mathrm{t})\right)$ where $\forall q \quad$ SOfferPart $_{\mathrm{q}} \in$ SharedOfferPart $_{\mathrm{a}}(\mathrm{t})$ and $p \neq q$;

25. $\quad$ if $\left(\mu_{\mathrm{i}}\left(\right.\right.$ SOfferPart $\left.\left._{\mathrm{p}}\right) \geq U_{i}^{t}\right)$ then

26. agent $i$ accepts SOfferPart ${ }_{\mathrm{p}}$; there is an agreement for the agents; break;

27. end if

28. $\quad r=r+1$;

29. end while

30. end if

31. $\quad i=i+1$;

32. end while

33. if (there is an agreement) then

34 break;

35. else

36. $\quad i=1$;

37. while $(i \leq N)$ do

38. $\quad U_{i}^{t+1}=U_{i}^{t}-\delta_{i}$;

39. if $\left(U_{i}^{t}<0\right)$ then $U_{i}^{t}=0$; end if

40. $\quad$ if $\left(U_{i}^{t}==0\right)$ then bSubmit=false; end if

41. $\quad \mathrm{i}=\mathrm{i}+1$;

42. end while

43. end if

44. $\mathrm{t}=\mathrm{t}+1$;

45. end while

Figure 2. The pseudo code of the negotiation process for $N$ agents. 
The computation complexity for the above protocol is $O\left(T \times N \times\left(T \times N \times N_{P A R} \times N_{L O C} \times N_{S T}\right)^{2}\right)$ where $T$ is the maximum number of rounds of the negotiation process; $N$ is the total number of agents; $N_{P A R}$ is the number of available choices of PAR; $N_{L O C}$ is the number of that for LOC; $N_{S T}$ is the number of that for ST. The exact computation time for finding an agreement is shown in Figure 12 at the end of Section Experiment. Given that the situations in our applications generally handle only several agents and a few typical issues with very limited choices, the computation time according to our experiment is acceptable.

\section{Theoretical Analysis towards the Negotiation Model}

As noted by Jennings et al. (2001) and Nisan et al. (2007), mechanism design involves the design of protocols for governing multi-agent interactions, such that these protocols have certain desirable properties, including Pareto optimal solutions, individual rationality, and guaranteed success.

\section{Pareto Optimal Solutions}

A solution $x \in X$ is Pareto efficient or Pareto optimal if there is no other solution $y \in X$ for which $\mu_{i}(y) \geq \mu_{i}(x)$ for all $i \in[1, N]$. Given an initial solution among a set of individuals, a change to a different solution that makes at least one individual better off without making any other individual worse off is called a Pareto improvement. A solution is defined as Pareto efficient or Pareto optimal when no further Pareto improvements can be made.

\section{Theorem 1}

The agreement achieved by our proposed protocol is Pareto efficient or Pareto optimal.

Proof:

Suppose our agreement is $x \in X$ where $x=\left\{x_{1}, \ldots, x_{V}\right\}, V$ is the total amount of issues, and there is another agreement, $y \in X$ where $y=\left\{y_{1}, \ldots, y_{V}\right\}$. According to our proposed protocol, the agreement $x$ is the first agreement encountered. Each individual gains his utility as denoted $\mu_{k}(x)$ and $k \in[1, N]$. The solution $y$ is another one encountered after $x$. The utilities for all the individuals are $\mu_{k}(y)$ and $k \in[1, N]$.

Because $y$ is encountered after $x$, based on our protocol in Figure 2, there must exist an agent $i$ and an agent $j$ such that $\mu_{i}(y)<\mu_{i}(x)$ while $\mu_{j}(y)>\mu_{j}(x)$ where $j \neq i$ and $j \in[1, N]$. Hence, according to the definition of Pareto improvement, $y$ is not a Pareto improvement on $x$. This situation is the same for any other agreement achieved after $x$. Therefore the solution $x$ is Pareto efficient or Pareto optimal because no further Pareto improvements can be made. 


\section{Individual rationality}

A model is said to be individually rational if following the protocol, i.e. "playing by the rules", is the best interests of negotiating participants. Individually rational models are essential because without them, there is no incentive for agents to engage in negotiations.

\section{Theorem 2}

The model proposed by us is individually rational.

Proof:

Because whether the outcome is an agreement or disagreement, every player gets a utility no less than zero, which is also the utility for every player if he does not participate in the negotiation. Therefore, the negotiation protocol is individually rational. $\square$

\section{Guaranteed success}

\section{Theorem 3}

The model proposed by us guarantees success if there is no time limit and a solution exists.

\section{Proof:}

If we assume that there is no agreement found, it means that the values of all the issues by one agent are not the same as the others. This may result when the deadline of the negotiation is too early. In contrast, our model guarantees success if there is no time limit. Hence it ensures that an agreement is certain to be reached if the utility functions for all or some of the agents share a common subset on all the issues, as shown by the pseudo code in Figure 2.

\section{Implication of the Negotiation Process}

There are essentially two basic approaches to model human beings' decision making process (Zeleny 1982). One is the outcome-oriented approach while another is the process-oriented approach. The rational (maximized social welfare where the sum of all the individuals' utilities is maximized) model is the conventional model in travel behavior analysis and this model is an outcome-oriented model. However individuals are not necessarily perfect utility maximizers. Our negotiation model is an attempt to better capture the process and thus to have a better basis for predicting outcomes correctly. In addition, utility maximization is very computationally demanding and becomes prohibitive in large-scale simulation systems. In contrast, the negotiation model is much more scalable especially when the number of issues in the decision problem becomes large.

\section{Experiment}

In this section, we demonstrate the negotiation procedure by employing the proposed negotiation approach between three agents with nonlinear utility functions as an example. The best choice for each agent on location, start time, duration, and participants is shown to be changed in the negotiation procedure. 


\section{Setting}

Assume there are three agents representing three friends to negotiate a joint shopping activity. They are interested in four issues: location, participants, start time, and the duration. In order to simplify the negotiation process, the deadline for all negotiators is 100 rounds. The maximum number of rounds here can be even decreased further so as to speed up the negotiation process, which will be discussed and shown in a later section (Comparison with Theoretical Results). The duration for going shopping should be within [0,9] hours. For the remaining issues, the agents have their own preferences, also in the range $[0,10]$. The acceptance utility level is decreased by 4.13 units per round. According to the utility curves shown in Figures 3, 4, and 5, the average of the maximum utility is about 413.0 units for agent 2 in Figure 4. Therefore, using this value of 4.13 per round, an agent can scan through all his utility curves after 100 rounds if necessary.

Agent 1 wants to go shopping with agent 2 and/or agent 3 together. His preferred stores/locations are V\&D, C\&A, or HEMA. Table 1 shows the preference values for each agent on the level of the parameters. In addition, the values of $\omega_{1}, \omega_{2}$, and $\omega_{3}$ are 10, 20, and 30, respectively. These values have been chosen for illustration purposes only, however, in applications they could be derived from data collected on people's preferences. As an example, agent 1's preference value for shopping location $\mathrm{V} \& \mathrm{D}$ is 5 and the travel time, $T T_{i, 1}$, to this location is 20 minutes which is denoted between brackets.

TABLE 1. Values for Three Parameters $\mathrm{ST}_{\mathrm{i}}, \mathrm{LOC}_{\mathrm{i}}$, and $\mathrm{PAR}_{\mathrm{i}}$

\begin{tabular}{|l|l|l|l|}
\hline Start time $\mathrm{ST}_{\mathrm{i}}$ & $\beta_{S T}^{1}$ & $\beta_{S T}^{2}$ & $\beta_{S T}^{3}$ \\
\hline 10 & 4 & 4 & $-\infty$ \\
\hline 12 & 8 & 4 & 3 \\
\hline 14 & 9 & 8 & 9 \\
\hline 16 & 4 & $-\infty$ & 1 \\
\hline 18 & $-\infty$ & $-\infty$ & 3 \\
\hline 20 & 4 & $-\infty$ & $-\infty$ \\
\hline 21 & 8 & $-\infty$ & $-\infty$ \\
\hline Location LCC $_{\mathrm{i}}$ & $\beta_{\text {LOC }}^{1}$ & $\beta_{\text {LOC }}^{2}$ & $\beta_{\text {LOC }}^{3}$ \\
\hline V\&D & $5(20$ minutes $)$ & $9(10$ minutes $)$ & $5(15$ minutes $)$ \\
\hline C\&A & $6(25$ minutes $)$ & $3(12$ minutes $)$ & $5(20$ minutes $)$ \\
\hline HEMA & $4(30$ minutes $)$ & $4(20$ minutes $)$ & $-\infty$ \\
\hline Participants PAR & $\beta_{P A R}^{1}$ & $\beta_{P A R}^{2}$ & $\beta_{P A R}^{3}$ \\
\hline $1,2,3$ & 8 & 6 & 5 \\
\hline 1,2 & 2 & 4 & 3 \\
\hline 1,3 & 4 & 0 & 9 \\
\hline
\end{tabular}




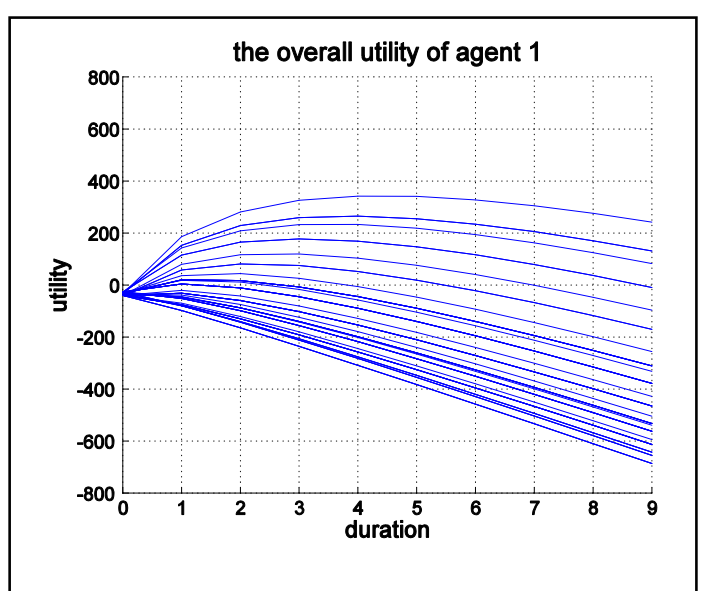

Figure 3. The utility curves of agent 1.

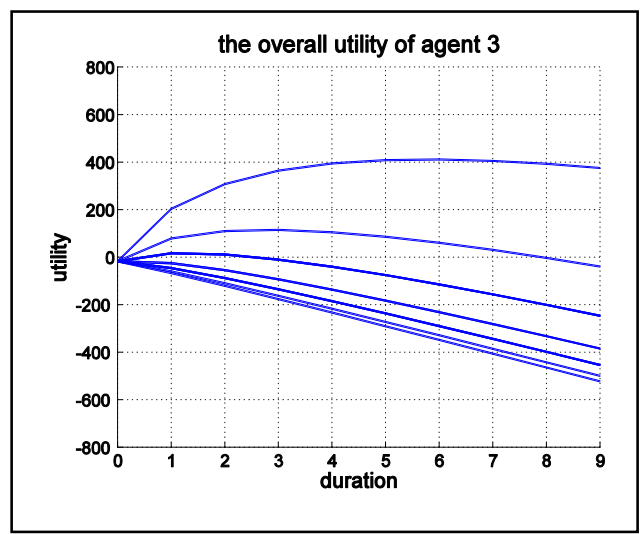

Figure 5. The utility curves of agent 3.

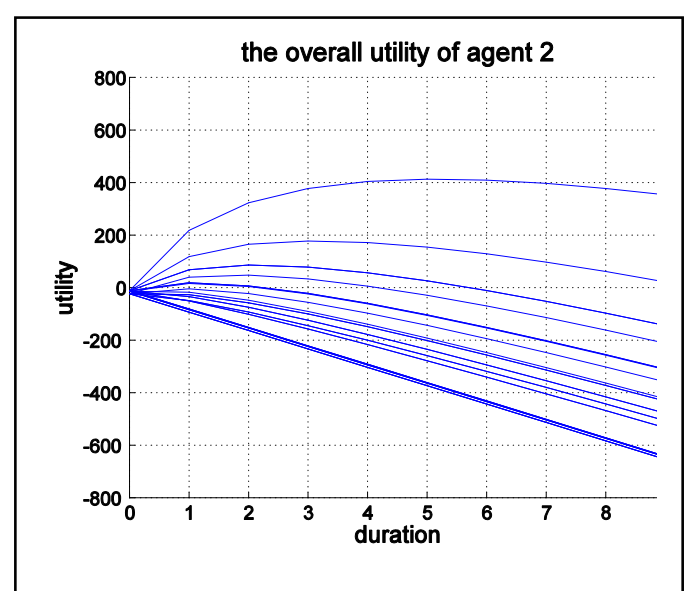

Figure 4. The utility curves of agent 2

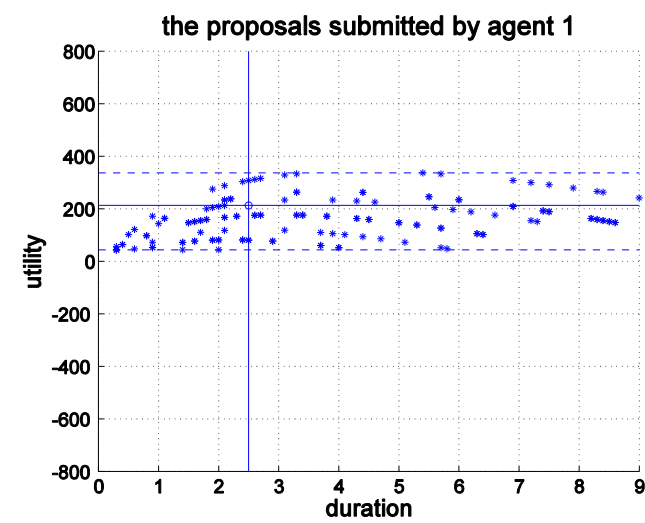

Figure 6. The proposals by agent 1.

\section{Result}

By employing the proposed model, agents 1,2, and 3 can efficiently generate and exchange their offers during the negotiation. All offers in each negotiation round are generated and displayed in Figures 6, 7, and 8, respectively.

In Figure 6, the dashed line on the top gives the initial acceptable utility level that can be achieved by agent 1 . The dashed line at the bottom gives the final acceptable utility level when the negotiation finishes. This is also shown for the other two agents in Figures 7 and 8. When there is an agreement for some or all agents, the offer agreed is pointed out by the cross point of a vertical line and a horizontal line with a small circle in the center.

In the first round, the first proposal submitted by agent 1 is shown by the star lying on the upper dashed line. When the other agents generate their proposals, they will first consider the proposals submitted by others before. If the proposals in the history of the process fall on their utility curves, then they compare whether those proposals give a higher utility than their acceptable utility level. If not, it means that those proposals can only give them less utility. Then they will submit their 
own proposals according to their best acceptable utility level. In our example, the first proposals are as follows:

- $\quad$ Agent 1 submits $\{14, \mathrm{C} \& A,\{1,2,3\}, 5.4$ hours $\}$;

- $\quad$ Agent 2 submits $\{14, \mathrm{~V} \& \mathrm{D},\{1,2,3\}, 5.2$ hours $\}$

- $\quad$ Agent 3 submits $\{14, \mathrm{~V} \& \mathrm{D},\{1,3\}, 6.8$ hours $\}$.

It is clear that there is no agreement at this moment: while the agents agree on start time, only 1 and 2 agree on the composition of the participants, only 2 and 3 agree on location, and there is no consensus regarding duration. (For durations to be considered as "equal" a margin of \pm 0.5 is allowed.)

In the future rounds, agent 1 will decrease his acceptable utility level step by step according to his concession strategy described above (in section Concession Strategy). Eventually, at round 71, when agent 3 decides on his proposals at this time, he checks the history of proposals and finds one offer shared by the other two agents: $\{14, \mathrm{~V} \& \mathrm{D},\{1,2,3\}, 2.5\}$. These two offers are $\{14$, $\mathrm{V} \& \mathrm{D},\{1,2,3\}, 2.1\}$ by agent 1 in round 30 and $\{14, \mathrm{~V} \& \mathrm{D},\{1,2,3\}, 2.5\}$ by agent 2 in round 14 . Agent 3 compares the utility brought by this proposal and his acceptable utility level and then chooses to follow this proposal because it brings him a higher utility. He does not choose to make this proposal earlier because at that time it does not return a higher utility than his own proposal.

The final agreement is shown in Figures 6, 7, and 8 by the intersection of two lines. The negotiation process comes to an end at round 71. It is shown that, in our experiment, the best choices for individual agents, i.e. Agent $1\{14, \mathrm{C} \& \mathrm{~A},\{1,2,3\}, 5.4\}$, Agent $2\{14, \mathrm{~V} \& \mathrm{D},\{1,2,3\}$, $5.2\}$, and Agent $3\{14, V \& D,\{1,3\}, 6.8\}$, have been changed to $\{14, V \& D,\{1,2,3\}, 2.5\}$ where the location is changed to $V \& D$, participant is changed to $\{1,2,3\}$; and the duration is changed to 2.5 hour.

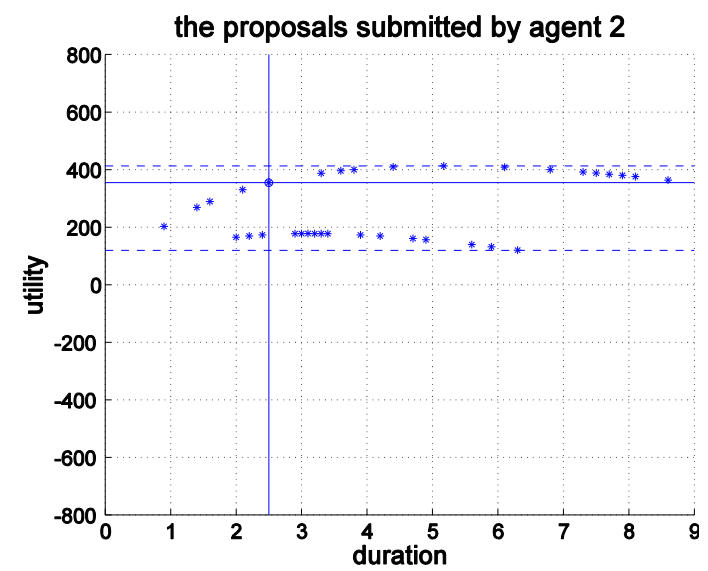

Figure 7. The proposals by agent 2.

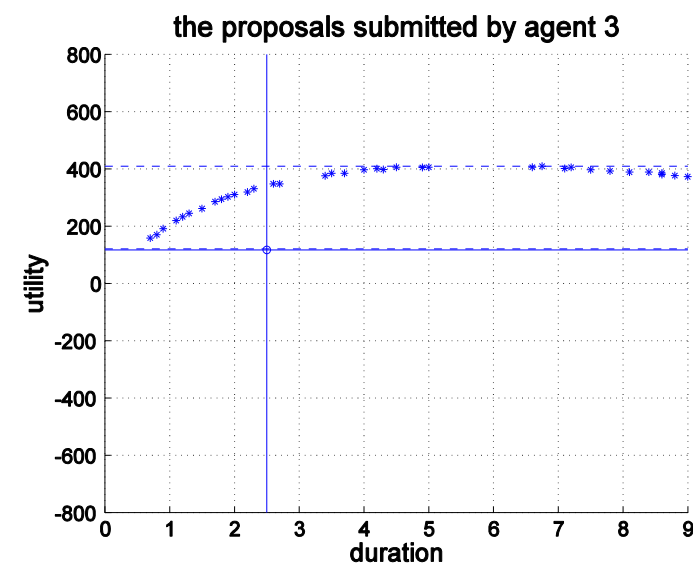

Figure 8. The proposals by agent 3. 


\section{Comparison with Theoretical Results}

In our case, social welfare refers to the sum of utilities across all the participants in the negotiation. The maximized social welfare solution (Andreu 1995) is the one which maximizes the sum of the utilities across all agents. Because our solution does not extensively search for all the possible agreements, the agreement achieved by our proposed algorithm does not guarantee to maximize social welfare. However it is useful to understand how close it is to the point of maximum social welfare or in other words the perfect situation. Therefore, we design the following experiment to determine the performance.

Besides that, the result achieved in the previous subsection is only for one run of the negotiation model. It will be more meaningful if we can determine the performance of our model on average across more runs. Hence the following experiment is necessary for a more thorough evaluation.

\section{Experiment Setup}

The setup is similar as before. However, in terms of number of rounds we impose a maximum of 10 rounds (instead of 100 rounds). A run, as before, is composed of multiple rounds. The change of the maximum number of rounds here is aimed at speeding up the negotiation process. The side effect is a decrease in the precision of the results.

To make the comparison for a range of utility function specifications, we carry out multiple runs while varying the values of parameters for each agent. There are multiple ways to vary the parameters in the utility functions, e.g., adjusting the values of $S T_{i}, L O C_{i}, P A R_{i}$, and $\omega$. We will impute random variation. Eventually 1000 runs will be carried out, which aims to give a stable result (He et al. 2003). In the experiment, we arbitrarily give agent 1, 2, and 3 the initial values of $\omega$ as $\omega_{1}=80, \omega_{2}=70$, and $\omega_{3}=60$.

In the first part of experimentation, we randomly draw values of $S T_{i}, L O C_{i}, P A R_{i}$ (see Table 1) and the value of $\omega$ at the beginning of each run. The range for each of these values is $[0,10]$ and a uniform probability distribution is assumed.

In the second part, we expect to observe some trend when the utility functions are changed in a specific systematic way. That is to say, it is interesting to change the cost per hour, $\omega$. In total, we conduct the experiment for 1000 runs with different values of $\omega$ for three agents which include 10 times making a change on $\omega_{1}$, another 10 times of making a change on $\omega_{2}$, and finally 10 times of making a change on $\omega_{3}$. We decrease monotonically the cost parameter by assuming that the step size, denoted as $\Delta$, is set to 6 . Then the values of the opportunity costs parameter is varied for the three agents as $\omega_{1}=80,74, \ldots, 26 ; \omega_{2}=70,64, \ldots, 16$; and $\omega_{3}=60,54, \ldots, 6$. 


\section{Result}

The result for the first part of the random changes on the values of preferences for $S T_{i}, L O C_{i}$, $P A R_{i}$, and the value of $\omega$ is shown in Figure 9. The $\mathrm{X}$ axis represents the serial number of runs from 1 to 1000. The $\mathrm{Y}$ axis is the ratio of the overall utilities for three agents achieved by our proposed protocol over that achieved by the maximized social welfare solution.

The total number of agreements out of 1000 runs/negotiations is 900 where 720 agreements shown by stars in the figure are achieved by three agents and the other 180 shown by circles in Figure 9 are achieved by two out of three agents. Moreover, the ratio of our Pareto optimal solution over the maximized social welfare solution by three agents is mostly more than 0.9 , which means that the outcome of our solution is quite close to that of the maximized social welfare solution. However, the location choice differs a lot between these two sets of solutions.

Compared to the agreements achieved by three agents, those achieved by two agents are very scattered if we look at all the small circles in Figure 9. The reason is that in case of a partial agreement, each agent chooses the agreement that he encounters first in his history list for any two participants (there will be more than one agreement) and this somewhat arbitrary choice will lead sometimes to a big difference between our solution and the maximized social welfare solution.

The remaining 100 unsuccessful runs are denoted by arrows at the bottom line of Figure 9. That is because there is no possible agreement first by all three agents and neither later by two agents.

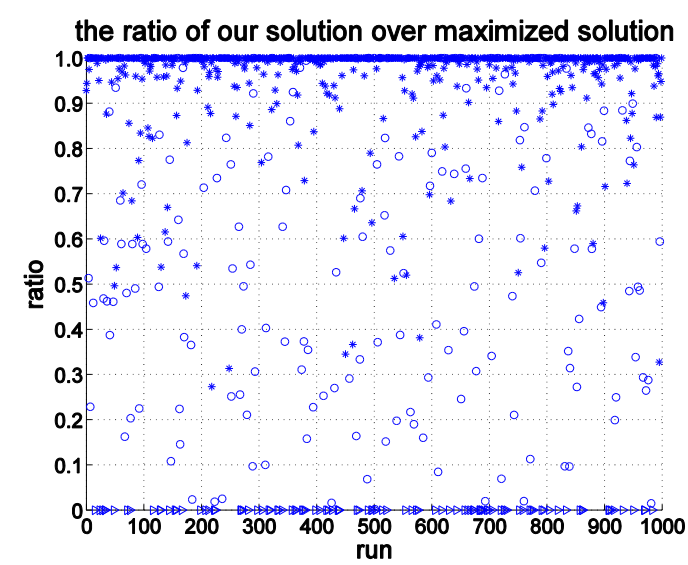

Figure 9. The ratio of the utilities gained by our Pareto optimal solution over the utilities gained by the maximized social welfare solution for three agents in a random parameter setting.

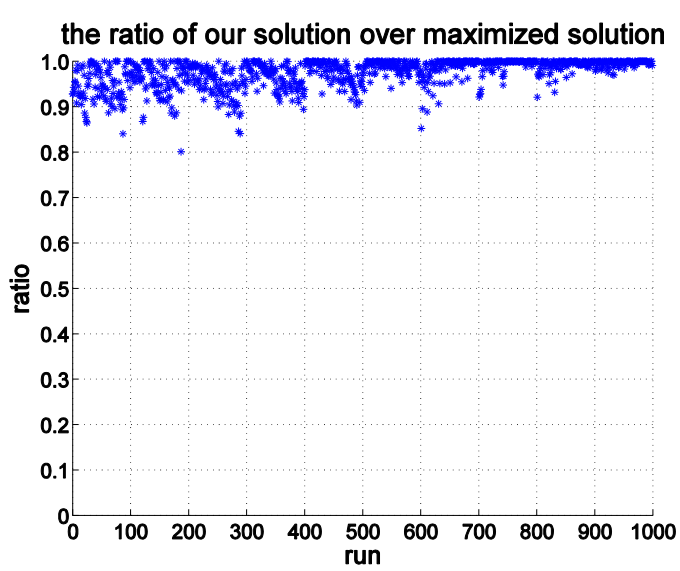

Figure 10. The ratio of utilities for three agents achieved by our model over that by the maximized social welfare solution for three agents in a systematic setting.

Figure 10 gives the result of the second part of experiments on achieving agreements in 1000 runs.

All these 1000 runs have their agreements. In particular, in most runs out of these 1000 runs/negotiations the ratio of our Pareto optimal solution and the maximized social welfare 
solution is more than 0.95 . The better performance in the second part compared with the first part of experiments is due to the smaller change of utility functions in the second part. On the one hand, all the three agents have valid utility curves higher than zero. On the other hand, there exists at least one solution shared by all the three agents according to their utility curves.

We can conclude from Figures 9 and 10 that our protocol performs very well in that it always finds an agreement when the deadline is long enough and when all the agents or part of the agents indeed share common values for all the parameters while their utilities are always non-negative. In the meanwhile the search for our Pareto optimal solution shown in Figure 11 can be finished much quicker than the search for the maximized social welfare solution. According to the figure, our solution costs about half of the time spent by the search for the maximized social welfare solution. The reason is that the latter one requires all the agents to go through all the concessions.

In addition, the execution time for finding an agreement in 1000 runs is given in Figure 12. According to Figure 12, the average execution time is about 0.2 seconds. Given this result, our model can meet the requirement of scalability and therefore be embedded in large scale agentbased simulation of dynamic joint activity scheduling.

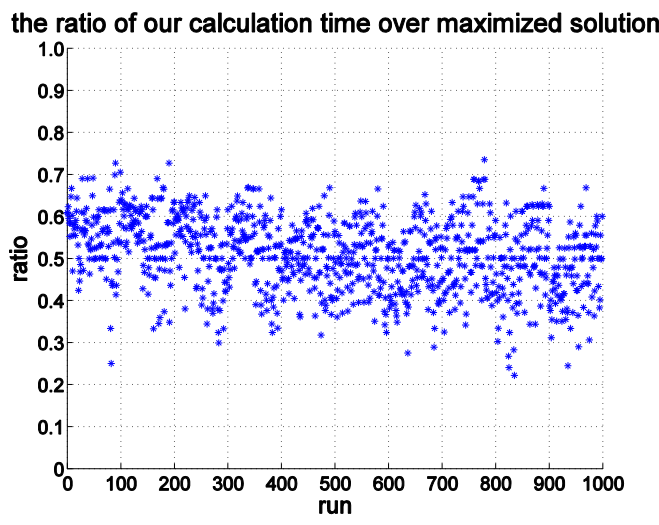

Figure 11. The ratio of calculation time for achieving an agreement by our model over that by the maximized social welfare solution in the same setting as Figure 10.

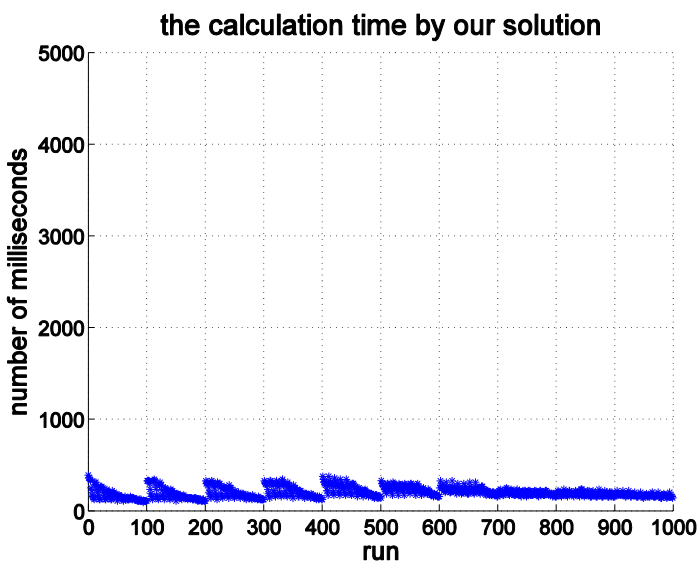

Figure 12. The exact calculation time for achieving an agreement by our model in the same setting as Figure 10.

\section{Discussion}

The proposed negotiation model is suitable to be applied in a more comprehensive activity scheduling system. On the one hand, the utility function used in the negotiation model takes care of the issues most likely to be considered in activity travel scheduling processes. On the other hand, in our multi-agent based dynamic activity-travel scheduling system (Ma et al. 2012), each agent is enabled to exchange messages so that negotiation can be realized to solve possible conflicts among agents. Therefore this system allows us to include certain aspects of social 
behavior of individuals which cannot be accommodated in existing utility-maximization systems. Within such a complicated environment, negotiation agents (i.e., agents who can negotiate through sending messages and making decisions.) will bring a big difference compared with other approaches to solve conflicts. For example, the proposed negotiation model can protect agents' private information from being disclosed to other agents, which simulates more of the real world. Moreover, agents can compromise and therefore have rights on determining their own pace of reaching an agreement. Several mechanisms which can speed up the negotiation procedure and further enhance realism, such as credit and power, have been discussed in Ma et al. (2011a, 2011b). We have also shown in the experiment section that our results are different compared to the maximized social welfare approach where the sum of utilities by all the agents is maximized.

There are also other issues related to activity-travel negotiation and scheduling processes, such as available transport modes, travel time, travel cost, etc. It is possible to follow the protocol of negotiation proposed in this work to negotiate these travel related issues as well. The most likely one in our application is to negotiate joint travel solutions separately where issues such as travel start time, travel duration, transport mode, travel cost, joint travel with other participants, and so on, can be handled more thoroughly. Since we focus on joint activity in this work, the issues discussed in our negotiation model are still limited given our focus.

As we have claimed in the section on the utility function, the parameters involved in our negotiation model can be estimated by conducting stated choice experiments. Moreover, other parameters, i.e., the range of deadlines of the negotiation process for each agent, such as the step size, $\delta$, during conceding, can be better investigated through questionnaires.

The negotiation protocol proposed in our work can handle one continuous attribute, i.e. the duration of the joint activity. If there are more than one continuous attributes, e.g. two, then the search space will be expanded to more dimensions, e.g., 3D. In that case, the difference in utility function between agents together with the number of continuous attributes will determine the shape of the search space and hence determine which search strategy is best for the complex search surface. The negotiation protocol and the search strategy rely a lot on the nature of the problem. Except this limitation, the number of discrete issues and number of agents can be less or more, which will not cause an extra burden to our proposed negotiation protocol. Moreover, the utility functions of different participants assumed in applications do not need to be the same as we have proposed in this study. The utility functions can be linear or nonlinear, monotonic or nonmonotonic, and continuous or discrete; all is up to the specific demands of an application.

\section{Conclusions and Future Work}

We have proposed a negotiation model for a semi-cooperative environment in which utility functions are nonlinear, non-monotonic, and private. Our approach searches the solution space by 
allowing agents to gradually concede utility whilst looking for a common solution. The solution achieved by our model is Pareto optimal; satisfies individual rationality; and our approach guarantees success.

In our application area of interest, the mechanism has been shown to be successful in finding an agreement for a shopping activity involving three people as a typical example. According to our experiments, our proposed mechanism can find the solution when the deadline is sufficiently long and when the solution is available in the utility functions of the participating agents. Given the computational performance, the model is applicable in large-scale multi-agent simulations. In the meanwhile, we have shown through the experiments that the location choices together with start time, participant, and duration are affected by the process of negotiation.

Since there is no universally best approach or technique for negotiation, it is worthwhile to explore and discuss other scenarios within the framework of our protocol. Our model can be easily extended to cases with other discrete issues in addition to the current issues, such as which day to carry out the shopping activity. Our proposed protocol is also possible to deal with cases that do not include continuous issues. Besides the current semi-cooperative environment, competitive environments for negotiation can be considered as well. Finally, the utility function can be different: linear or nonlinear, monotonic or non-monotonic, and continuous or discrete.

Future work on our agenda includes integrating the negotiation procedure into a larger scale agentbased simulation of activity-travel behavior. In addition, the data collection is ongoing. After the data collection is finished, empirical estimation of the parameters and validation need to be conducted in future research.

\section{Acknowledgments}

The research leading to these results has received funding from the European Research Council under the European Community's Seventh Framework Programme (FP7/2007-2013) / ERC grant agreement no. 230517 (U4IA project).

The views and opinions expressed in this publication represent those of the authors only. The ERC and European Community are not liable for any use that may be made of the information in this publication.

\section{References}

Andreu Mas-Colell, Whinston Michael Dennis, Green Jerry R. (1995) Microeconomic theory. Oxford University Press.

Arentze T.A., Timmermans H.J.P. (2007) Modelling dynamics of activity-travel behaviour. In: Proceedings $12^{\text {th }}$ HKSTS Conference, Hong Kong. 
Arentze T.A., Timmermans H.J.P. (2009) A need-based model of multi-day, multi-person activity generation. Transportation Research B 43(2): 251-265.

Arentze T.A., Pelizaro C., Timmermans H.J.P. (2010) An agent-based micro-simulation framework for modeling of dynamic activity-travel rescheduling decisions. International Journal of Geographical Information Science. 24(8): 1149-1170.

Avineri E. (2006) Measuring and simulating altruistic behaviour in group travel choice decisions. In: Proceedings of the 11th International Conference on Travel Behaviour Research.

Balmer M. (2007) Travel demand modeling for multi-agent transport simulations: algorithms and systems. Ph.D. dissertation, ETH Zurich.

Becker Gary S. (1965) A theory of the allocation of time. The Economic Journal 75(299): 493517.

Ettema D.F., Arentze T.A., Timmermans H.J.P. (2007) Social influences on household location, mobility and activity choice in integrated micro-simulation models. In: Proceedings of the Workshop on Frontiers in Transportation, Amsterdam.

Fang, Z., Tu, W., Li, Q. and Li, Q., (2011). A multi-objective approach to scheduling joint participation with variable space and time preferences and opportunities. Journal of Transport Geography 19(4), 623-634.

Fatima Shaheen S., Wooldridge Michael, Jennings Nicholas R. (2004) An agenda-based framework for multi-issue negotiation. Artificial intelligence 152(1):1-45.

Gliebe J.P., Koppelman F.S. (2002) A model of joint activity participation between household members. Transportation 29(1): 49-72.

Gilbert Nigel (2008) Agent-based models (quantitative applications in the social sciences). Sage Publications.

Grigolon Anna B., Kemperman Astrid D.A.M., Timmermans Harry J.P. (2012) The influence of low-fare airlines on vacation choices of students: Results of a stated portfolio choice experiment, Tourism Management, 33(5): 1174-1184.

Hackney J., Marchal F. (2009) A model for coupling multi-agent social interactions and traffic simulation. In: Proceedings of the Transportation Research Board $88^{\text {th }}$ Annual Meeting.

He M., Leung H., Jennings N. R. (2003) A fuzzy logic based bidding strategy for autonomous agents in continuous double auctions. IEEE Transactions on Knowledge and Data Engineering 15(6): 1345-1363.

Hensher David A., Rose John M., Greene William H. (2005) Applied choice analysis: a primer. Cambridge University Press.

Jacques Wainer, Paulo Roberto, Everton Rufino Constantino (2007) Scheduling meetings through multi-agent negotiations. Decision Support Systems 44(1): 285-297.

Jennings N.R., Faratin P., Lomuscio A.R., Parsons S., Wooldridge M.J., Sierra C. (2001)

Automated negotiation: prospects, methods and challenges. Group Decision and Negotiation 10(2): 199-215.

Kuwano M., ZHANG J., FUJIWARA A. (2011) A dynamic discrete choice model with multidimensional social interactions. In: Proceedings of the Transportation Research Board $90^{\text {th }}$ Annual Meeting. 
Ma Huiye, Ronald Nicole, Wu Mengxiao, Arentze Theo, Timmermans Harry (2010) Multi-player Multi-issue negotiation with incomplete information in agent-based activity-travel scheduling. In: Proceedings of the 10th International Conference on Design and Decision Support Systems in Architecture and Urban Planning.

Ma Huiye, Arentze Theo, Timmermans Harry (2012) An agent based model of dynamic activitytravel scheduling and implementation. In: Proceedings of the Transportation Research Board $91^{\text {st }}$ Annual Meeting.

Ma Huiye, Nicole Ronald, Arentze Theo, Timmermans Harry (2011a) A New Credit Mechanism for Semicooperative Agent-Mediated Joint Activity-Travel Scheduling: Negotiating with Incomplete Information. Transportation Research Record Issue 2230: 104-110.

Ma Huiye, Nicole Ronald, Arentze Theo, Timmermans Harry (2011b) Incorporate power into joint activity-travel scheduling. In: Proceedings of $16^{\text {th }}$ HKSTS Conference, Hong Kong.

Osborne M., Rubinstein A. (1994) A course in game theory. The MIT Press.

Neutens, T., Schwanen, T. and Miller, H. J., (2010). Dealing with timing and synchronization in opportunities for joint activity participation. Geographical Analysis 42(3), 245-266.

Nijland, Linda , Arentze, Theo, Borgers, Aloys, and Timmermans, Harry (2011) Modelling Complex Activity-Travel Scheduling Decisions: Procedure for the Simultaneous Estimation of Activity Generation and Duration Functions, Transport Reviews 31(3): 399-418.

Nisan Noam, Roughgarden Tim, Tardos Eva, Vazirani, Vijay V. (2007) Algorithmic game theory. Cambridge University Press.

Ren Fenghui, Zhang Minjie, Fulcher John (2010) Bilateral single-issue negotiation model considering nonlinear utility and time constraint. In: Proceedings of the Third International Workshop on Agent-based Complex Automated Negotiations.

Rindt Craig R., Marca James E., McNally Michael G. (2003) An agent-based activity microsimulation kernel using a negotiation metaphor. In: Proceedings of the Transportation Research Board $82^{\text {nd }}$ Annual Meeting.

Ronald N., Arentze T.A., Timmermans H.J.P. (2009). Modelling social interactions between individuals for joint activity-travel scheduling". In: Proceedings of the 11th International Conference on Travel Behaviour Research.

Ronald N., Dignum Virginia, Jonker Catholijn (2010) When will I see uou again: modeling the influence of social networks on social activities. In: Proceedings of the Multi-Agent Logics, Languages, and Organisations Federated Workshops.

Rubinstein Ariel. (1985) A bargaining model with incomplete information about time preferences. Econometrica 53(5):1151-1172.

Sandholm Tuomas, Nir Vulkan Y. (1999) Bargaining with deadlines. In: Proceedings of Association for the Advancement of Artificial Intelligence.

Scott D.M., Kanaroglou P.S. (2002) An activity-episode generation model that captures interactions between household heads: development and empirical analysis. Transportation Research B 36(10): 875-896. 
Takayuki Ito, Hiromitsu Hattori, Mark Klein. (2007) Multi-issue negotiation protocol for agents: exploring nonlinear utility spaces. In: Proceedings of International Joint Conference on Artificial Intelligence pp. 1347-1352.

van den Berg, P.E.W., Arentze, T.A., Timmermans, H.J.P. (2012) A multilevel path analysis of contact frequency between social network. Journal of Geographical Systems 14(2): 125-141.

Weiss Gerhard (1999) Multiagent systems: a modern approach to distributed artificial intelligence. The MIT Press.

Wu Mengxiao, de Weerdt Mathijs, La Poutre Han (2009) Efficient methods for multi-agent multiissue negotiation: allocating resources. In: Proceedings of Principles of Practice in Multi-Agent Systems pp. 97-112.

Yaron Hollander (2006) The applicability of non-cooperative game theory in transport system analysis. In: Proceedings of the Transportation Research Board $85^{\text {th }}$ Annual Meeting. Zeleny M. (1982) Multiple Criteria Decision Making. McGraw-Hill Book Company. Zhang Junyi, Timmermans H.J.P., Borgers A. (2005) A model of household task allocation and time use. Transportation Research B 39(1): 81-95. 


\section{University Library}

\section{- M M N E R VA A gateway to Melbourne's research publications}

Minerva Access is the Institutional Repository of The University of Melbourne

Author/s:

Ma, H;Ronald, N;Arentze, TA;Timmermans, HJP

Title:

Negotiating on location, timing, duration, and participant in agent-mediated joint activitytravel scheduling

\section{Date:}

2013-01-01

\section{Citation:}

Ma, H., Ronald, N., Arentze, T. A. \& Timmermans, H. J. P. (2013). Negotiating on location, timing, duration, and participant in agent-mediated joint activity-travel scheduling. Journal of Geographical Systems, 15 (4), pp.427-451. https://doi.org/10.1007/s10109-012-0173-0.

Persistent Link:

http://hdl.handle.net/11343/282874 